\title{
The Efficacy of Bronchoscopy versus Computerised Tomography in Initial Identification of Patients with Hemoptysis
}

\author{
Dingxiu $\mathrm{He}^{1,2}$, Dong Huang ${ }^{2}$, Kaisen Huang ${ }^{3}$ and Zongan Liang ${ }^{2}$ \\ ${ }^{1}$ Department of Emergency, The People's Hospital of Deyang, Sichuan, China \\ ${ }^{2}$ Department of Respiratory and Critical Care Medicine, West China Hospital, Sichuan University, Sichuan, China \\ ${ }^{3}$ Department of Cardiology, The People's Hospital of Deyang, Sichuan, China
}

\begin{abstract}
The effects of bronchoscopy and chest CT on early evaluation of patients with hemoptysis are still controversial. PubMed, EMBASE, and the Cochrane Library databases were systematically searched. Odds ratio (OR) was applied to assess the utility of bronchoscopy for hemoptysis etiology and site in comparison with CT in the various clinical processes. A total of 23 studies were included ( $\mathrm{N}=4635)$. The results showed that bronchoscopy implied a lower overall diagnostic accuracy, especially in identifying the etiology of hemoptysis, compared with $\mathrm{CT}(\mathrm{OR}=0.34,95 \% \mathrm{Cl}:[0.23,0.51], \mathrm{OR}=0.21,95 \% \mathrm{Cl}:[0.14,0.31]$, respectively). When the results of radiograph were normal, the effectiveness of bronchoscopy was significantly weaker than that of CT $(\mathrm{OR}=0.32,95 \% \mathrm{Cl}:[0.22,0.45])$. In the cases of massive hemoptysis, bronchoscopy and CT had no statistical significance for identifying bleeding $(\mathrm{OR}=0.27,95 \% \mathrm{Cl}:[0.02,3.18])$. The study suggested that bronchoscopy did not show superior diagnostic accuracy than $\mathrm{CT}$ for patients with hemoptysis at the first visit.
\end{abstract}

Key Words: Hemoptysis, Bronchoscopy, CT, Meta-analysis.

How to cite this article: He D, Huang D, Huang K, Liang Z. The Efficacy of Bronchoscopy versus Computerised Tomography in Initial Identification of Patients with Hemoptysis. J Coll Physicians Surg Pak 2021; 31(12):1459-1467.

\section{INTRODUCTION}

Hemoptysis is defined as bleeding originating from the lung alveoli or airways of the lower respiratory tract, ${ }^{1}$ which is a common and challenging symptom that accounts for $0.2 \%$ of all hospitalised patients. ${ }^{2}$ However, there are a wide spectrum and variations in etiology reported according to the time of publication, geographic location, and medical care facility. ${ }^{3-5}$ It is crucial to manage patients with hemoptysis depending upon initial identification of the etiology and localisation of the bleeding.

Bronchoscopy is the main procedure of choice, which plays a key role in detecting the etiology and the sites of bleeding. Naidich et al. explained that bronchoscopy outlined the exact location and submucosal extension of tumors. ${ }^{6}$ Indeed, bronchoscopy could better assess upper airways and endobronchial abnormalities and could provide histopathological and microbiological samples. ${ }^{7}$ However, other studies showed that bronchoscopy could not localise the bleeding site and cause as effectively as compared with CT. ${ }^{8,9}$

Correspondence to: Zongan Liang, Department of Respiratory and Critical Care Medicine, West China Hospital, Sichuan University, Sichuan, China

E-mail: liangza@scu.edu.cn

Received: April 27, 2021; Revised: August 29, 2021;

Accepted: October 16, 2021

DOI: https://doi.org/10.29271/jcpsp.2021.12.1459
Patients with hemoptysis may seek treatment in the Emergency Department or visit general practice or respiratory medicine. ${ }^{10,11}$ The strategy for the investigation of patients with hemoptysis remains underdiscussion. It is important to decide which procedure to adopt first, in assessing hemoptysis.

Therefore, the purpose of this review was to evaluate the optimal timing of bronchoscopy in a series of patients presenting with hemoptysis.

\section{METHODOLOGY}

This review was conducted according to the series of patients with hemoptysis visits. Figure 1 shows the patient's initial evaluation flow framework and key questions that guided the review.

KQ1. Should the bronchoscopy be used as routine workup to screen all patients with hemoptysis?

KQ 1a. Should the bronchoscopy be used as a location strategy? KQ 1b. Should the bronchoscopy beused as a cause strategy?

KQ 2. Should the bronchoscopy be used for all patients with normal radiographs?

KQ 3. How does bronchoscopy contribute to the diagnosis with negative findings on $\mathrm{CT}$ ?

KQ 4. Should the bronchoscopy be used to routinely investigate massive hemoptysis patients?

KQ 5. Should the bronchoscopy be used to screen all hemoptysis with a high risk of malignancy? 


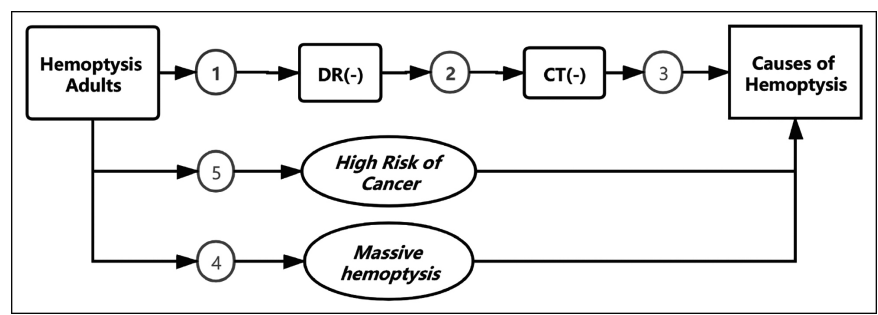

Figure 1: Patient pathway flow framework and key questions (KQs).

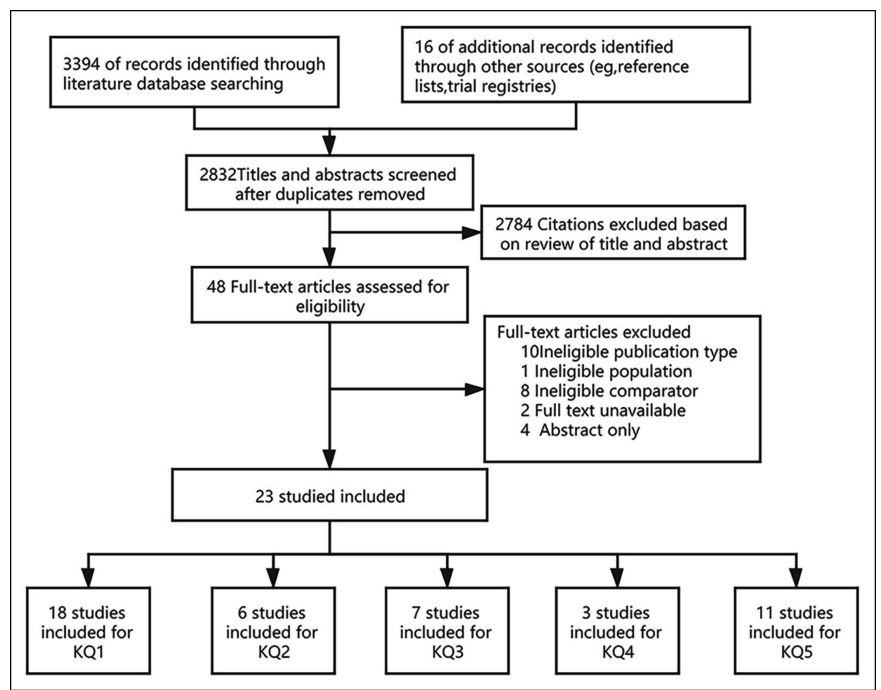

Figure 2: Summary of evidence search and selection.

A systematic and comprehensive search was conducted in these databases: Embase, PubMed and Cochrane Library to December 1, 2020. The search terms included "hemoptysis" OR "airway bleeding" OR "pulmonary bleeding", "bronchoscope" OR "bronchoscopy" OR "fiberoptic bronchoscopy" OR "FOB"; and "tomography, X-ray computed" OR "computed tomography" OR "CT". ClinicalTrials.gov and Cochrane databases were also searched for unpublished literature. All the reference lists of the included articles and review articles about hemoptysis were also selected and evaluated. The last surveillance was conducted on December 12, 2020.

Two investigators (HDX and HKS) independently conducted the reviews of the titles, abstracts, and full-text articles to determine eligibility, using a common set of criteria for each key question (KQ). The divergence in opinions was resolved through discussion or with the help of a third reviewer (HD). The review included studies that included: patients presenting with hemoptysis older than 16 years, and both CT and bronchoscopy were compared. The following exclusion criteria were used: Studies including patients with previous known diagnosis of disease relative to hemoptysis; incomplete or duplicated data; letters, case reports or review articles.

For massive hemoptysis, the cutoff value ranged from 100 to $600 \mathrm{ml}$ of blood produced in 24 hours. ${ }^{12,13}$ Physicians only estimate the volume and do not know the real volume. For this review, the definitions of massive hemoptysis that the included studies used, were accepted.

To avoid overlapping patient populations, the data were compared via recruitment years and data sources. If a patient population was found to overlap, only the article with the most comprehensive population was included. This resulted in the exclusion of one article from this systematic review. ${ }^{14}$ For each included study, one investigator (HDX) extracted the information about the first author; publication year; designs of the studies; characteristics and demographics, sample size, duration of research and follow-up; causes of hemoptysis; and comparators and outcomes. The second investigator (HKS) checked the results for completeness and accuracy.

Two independent investigators (HDX and HD) assessed the quality of each included study, using the Newcastle-Ottawa Scale (NOS). The cross-sectional study, as a modified version of the case-control study criteria, got a maximum score of 7. Scores $\leq 5$ were considered low quality. Disagreements were resolved by discussion and consensus.

Findings for each question weresummarised intabularand narrative form. Forthe meta-analysis, odds ratios(ORs) with $95 \%$ confidence intervals ( $95 \% \mathrm{Cls}$ ) by forest plot for dichotomous comparisons across all studies were pooled. Heterogeneity between studies was assessed using Cochran's Q test and Higgins I'statistics. $P<0.1$ or $I^{2} \geq 50 \%$ was defined as high heterogeneity; then, the effect size by means of random or fixed models for heterogeneous or homogeneous studies were estimated, respectively. A subgroup analysis was also performed to determine whether the study design affected the results of this study. A sensitivity analysis was performed by removing the data of each individual study in turn each time. Potential publication bias was evaluated by the Begg funnel plot. Statistical analysis was performed using Review Manager (version 5.4). Statistical significance was rendered as $p<0.05$.

\section{RESULTS}

The literature search yielded 3,394 articles and 22 articles through the references; of which, 23 studies $^{3-5,7-9,15-30}$ met the inclusion criteria for the overall systematic review of the comparison of bronchoscopy and chest CT imaging (Figure 2).

The 23 eligible studies were published between 1990 and 2020 and included a total of 4,635 participants from 13 countries (Table I). The majority of participants were males (52.6\%-91\%) and current or former smokers (24-91\%). The main causes included malignancy (0.4-41.1\%), bronchiectasis (2-57.1\%), pneumonia or airway infection (3.2-69\%), and tuberculosis (0.6-50.9\%). Cryptogenic hemoptysis account for $5.4-83.8 \%$.

Meta-analysis was performed to evaluate the effectiveness of bronchoscopy for hemoptysis etiology and bleeding site detection in comparison with $\mathrm{CT}$, and the main results for each key question are summarised below (Table II).

In general, compared with $\mathrm{CT}$, bronchoscopy implied an initial overall effectiveness, especially regarding identification of the etiology of hemoptysis ( $O R=0.34,0.21$, respectively). Nosignificant difference in finding the location of hemorrhage was noted forbronchoscopy and CT (OR=0.00, Figure 3). 
Table I: Main characteristics of studies included.

\begin{tabular}{|c|c|c|c|c|c|c|c|c|c|c|c|c|c|c|c|}
\hline Study & Type & Location & $\begin{array}{l}\text { Research } \\
\text { time }\end{array}$ & Follow-up & $\begin{array}{c}\text { Patients } \\
\text { (n) }\end{array}$ & $\begin{array}{c}\text { Men,n } \\
(\%)\end{array}$ & Age (years) & $\begin{array}{c}\text { Smokers } \\
\mathrm{n}(\%)\end{array}$ & Amount & Malignancy,n(\%) & $\begin{array}{c}\text { Bronchiectasis, } \\
\mathrm{n}(\%)\end{array}$ & Infection,n(\%) & $\mathrm{TB}, \mathrm{n}(\%)$ & $\begin{array}{c}\text { Cryptogenic, } \\
\mathrm{n}(\%)\end{array}$ & $\begin{array}{c}\begin{array}{c}\text { Quality } \\
\text { assessment } \\
\text { (NOS) }\end{array} \\
\end{array}$ \\
\hline Naidich-1990 $0^{15}$ & Retro & USA & 1988.4-1989.7 & & 58 & $50 \square 86 \square$ & 56 & NA & NA & $24(41.4)$ & $10(17.2)$ & NA & NA & NA & 6 \\
\hline Patricia-1993 ${ }^{16}$ & Pros & England & $\mid 1991.3-1992.12$ & & 91 & $64 \square 70 \square$ & 63.14 & 79(87) & Mild-moderate & $35(38.4)$ & $14(15.4)$ & $3(3.2)$ & NA & NA & 6 \\
\hline McGuinness-1994 & Pros & USA & $|1991.7-1992.4|$ & & 57 & $47(82)$ & $59(26-74)$ & $49(86)$ & Mild-moderate & $7(12)$ & $14(25)$ & $4(7)$ & $9(16)$ & $11(19)$ & 6 \\
\hline Hirshberg-1997 $7^{18}$ & Retro & Israel & $\mid 1980.1-1995.8$ & & 208 & $127 \square 61 \square$ & $58 \pm 17$ & $110(53)$ & ALL & 39(19) & $41(20)$ & $70(34)$ & $3(1.4)$ & $17(8 \square$ & 6 \\
\hline Tak-1999 ${ }^{3}$ & Pros & India & NA & & 50 & $33(66)$ & $37.2(15-68)$ & $12(24)$ & Mild-moderate & $3(6)$ & $12(24)$ & 1(2) & 1(2) & $33(66)$ & 7 \\
\hline Hsiao-2001 ${ }^{19}$ & Retro & USA & $1988-2000$ & & 28 & 19(68) & 54.6ロ16-91) & NA & Massive & $4(14.3)$ & $16(57.1)$ & 0 & $2(7.1)$ & $2(7.1)$ & 6 \\
\hline Abal-2001 ${ }^{20}$ & Pros & Kuwait & $\begin{array}{l}1998.1- \\
1998.11\end{array}$ & $1 y$ & 52 & $42(81)$ & $42.4(16-86)$ & $31(60)$ & ALL & $5(9.6)$ & $11(21.2)$ & $3(5.8)$ & $17(32.7)$ & $13(25)$ & 7 \\
\hline Fidan-2002 & Retro & Turkey & $2000.1-2000.12$ & & 108 & 79(75) & $51.74 \pm 17.51$ & $65(60)$ & ALL & $37(34.3)$ & $27(25)$ & $11(10.2)$ & $|19(17.6)|$ & $9(8.3)$ & 6 \\
\hline Revel-2002 $2^{21}$ & Retro & USA & 1995.1- 1999.6 & & 80 & $57(71)$ & 58ロ20-93) & NA & Massive & $9(11)$ & $25(31)$ & $8(10)$ & $15(19)$ & $8(10)$ & 7 \\
\hline Tsoumakidou-2006 $6^{22}$ & Pros & Greece & $\begin{array}{l}2001.1- \\
2003.11 \\
\end{array}$ & $2-4 y$ & 168 & $137(81)$ & NA & $145(86)$ & ALL & $24(13)$ & $48(26.1)$ & $35(19)$ & $8(4.3)$ & $10(5.4)$ & 7 \\
\hline Khalil-2007 & Retro & France & 2year-period & & 80 & $67(84)$ & $56(28-86)$ & NA & ALL & $4(5)$ & $23(28.7)$ & $1(1.25)$ & 19(23.7) & $27(33.7)$ & 6 \\
\hline Thirumaran-2009 $9^{23}$ & Retro & UK & 2001.3-2005.12 & & 270 & $162(60 \%)$ & 60 & $246(91)$ & NA & $26(9.6)$ & $20(7.3)$ & $16(69)$ & $4(1.5)$ & $16(5.8)$ & 7 \\
\hline Uzun-2010 & Pros & Turkey & 2003.11-2006.9 & & 178 & $136(76 \%)$ & $54.3 \pm 16$ & 119(66.9) & ALL & $53(29.7)$ & $23(12.9)$ & $14(7.8)$ & $11(6.2)$ & $10(5.6)$ & 7 \\
\hline Lee-2012 $2^{24}$ & Retro & Korea & 2003.1-2009.10 & $2.1 y$ & 228 & $120(52.6)$ & 51.6 & $98(43.0)$ & $\mathrm{NA}$ & $1(0.4)$ & $\mathrm{NA}$ & $9(3.9)$ & NA & 191(83.8) & 7 \\
\hline Mohammad- $2015^{25}$ & Retro & Iran & NA & & 40 & $22(55)$ & $44(22-77)$ & NA & NA & $2(5 \%)$ & $11(27.5)$ & $3(7.5)$ & $6(15)$ & $8 \square 20 \square$ & 5 \\
\hline Bønløkke-2015 & Retro & Denmark & $2000 \quad 2010$ & $2 y$ & 269 & 159(59.0) & $55.4 \pm 15.3$ & $\mathrm{NA}$ & Mild-moderate & $16 \square 6.0 \% \square$ & 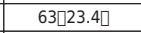 & NA & $\mathrm{NA}$ & $\mathrm{NA}$ & 6 \\
\hline Seon-2016 $6^{26}$ & Retro & South Korea & 2005.1-2009.7 & & 161 & 94 (58) & $57(48-68)$ & NA & ALL & $4(2.5 \%)$ & $36(22.4)$ & $11(6.8)$ & $31(19.3)$ & $36(22.4)$ & 7 \\
\hline Nielsen-2016 ${ }^{27}$ & Retro & Italy, Denmark & 2009.1-2014.11 & & 326 & $206(63)$ & $60.5 \square 15.3$ & $262(80)$ & mild-moderate & $13(4.0 \%)$ & $19(5.8)$ & $53(16.3)$ & $2(0.6)$ & $171(52.5)$ & 6 \\
\hline muhammad-2017 ${ }^{28}$ & Retro & India & 1 year & & 175 & $160(91)$ & $57.31 \pm 13.57$ & NA & ALL & $54(33.72 \%)$ & NA & NA & $84(50.9)$ & NA & 6 \\
\hline Arooj-2018(1) & Retro & Ireland & 2011-2012 & & 155 & $82(53)$ & $59 \pm 12.2$ & NA & NA & $24(16 \%)$ & $3(2)$ & $73(47)$ & NA & $25(16)$ & 6 \\
\hline Arooj-2018(2) ${ }^{5}$ & Pros & Ireland & 2013-2016 & $6 \mathrm{~m}$ & 182 & \begin{tabular}{|l|}
$116(64)$ \\
\end{tabular} & $61 \pm 10.2$ & NA & $\mathrm{NA}$ & $33(18 \%)$ & $17(9)$ & $91(50)$ & NA & $35(19)$ & 7 \\
\hline Mondoni-2019 $9^{29}$ & Retro & italy & 2013.7_2015.9 & & 486 & $336(69)$ & 67ロ53-76口 & $327(54)$ & ALL & NA & NA & NA & NA & NA & 6 \\
\hline christian-202030 & Retro & Denmark & 2006.1-2016.11 & & 1185 & 726(61) & $57.5 \pm 14.55$ & 871ロ74ロ & NA & 0 & $26(2.2)$ & $149(12.6)$ & $\mathrm{NA}$ & 989(83.5) & 6 \\
\hline
\end{tabular}

Table II: Summary of evidence for evaluation of hemoptysis.

\begin{tabular}{|l|c|c|c|}
\hline $\begin{array}{l}\text { Key question and } \\
\text { topic }\end{array}$ & $\begin{array}{c}\text { No. of } \\
\text { studies }\end{array}$ & $\begin{array}{c}\text { No. of } \\
\text { participants }\end{array}$ & $\begin{array}{c}\text { Summary of main } \\
\text { findings }\end{array}$ \\
\hline $\begin{array}{l}\text { KQ1: Initial } \\
\text { evaluation }\end{array}$ & 18 & 3472 & $\begin{array}{c}\mathrm{OR}=0.34,95 \% \mathrm{Cl}:[0.23, \\
0.51, p<0.001, I^{2}=90 \%\end{array}$ \\
\hline KQ1a: Bleeding site & 6 & 847 & $\begin{array}{c}\mathrm{OR}=0.00,95 \% \mathrm{Cl}:[-0.04, \\
0.05], p=0.47, I^{2}=0 \%\end{array}$ \\
\hline $\begin{array}{l}\text { KQ1b: Bleeding } \\
\text { cause }\end{array}$ & 13 & 1374 & $\begin{array}{c}\mathrm{OR}=0.21,95 \% \mathrm{Cl}:[0.14, \\
0.31], p<0.001, I^{2}=75 \%\end{array}$ \\
\hline KQ2: DR negative & 6 & 401 & $\begin{array}{c}\text { OR=0.32, 95\% Cl: [0.22, } \\
0.45], p=0.23, I^{2}=27 \%\end{array}$ \\
\hline KQ3: CT negative & 7 & 738 & $\begin{array}{c}\text { Only } 4 \text { meaningful positive } \\
\text { cases }\end{array}$ \\
\hline $\begin{array}{l}\text { KQ4: Massive } \\
\text { hemoptysis }\end{array}$ & 3 & 123 & $\begin{array}{c}\text { OR=0.27, 95\% Cl: }[0.02, \\
3.18], p=0.001, I^{2}=87 \%\end{array}$ \\
\hline $\begin{array}{l}\text { KQ5: High risk of } \\
\text { cancer }\end{array}$ & 11 & 175 & $\begin{array}{c}\text { OR=0.12, 95\% Cl: [0.05, } \\
0.28], p=0.76, I^{2}=0 \%\end{array}$ \\
\hline
\end{tabular}

When the radiograph was normal, the effectiveness of bronchoscopy was significantly weaker than that of CT (OR = 0.32 , Figure 4).

Among the 738 patients with a negative $\mathrm{CT}$, bronchoscopy only selected 4 tumor patients. Hirshberg et al. ${ }^{18}$ from Jerusalem, Israel reported that CT alone failed to locate three lung cancers that were successfully found by bronchoscopy. Lee et al. showed that only one in 228 patients was diagnosed with malignancy by initial bronchoscopy. ${ }^{24}$
In massive hemoptysis, no statistical significance was noted between bronchoscopy and CT (OR $=0.27,95 \% \mathrm{Cl}$ : [0.02, 3.18], supplementary Figure 5).

In the screening of hemoptysis with a high risk of lung cancer, bronchoscopy was dramatically weaker than CT $(\mathrm{OR}=0.12$, supplementary Figure 6$)$.

Significant heterogeneity was noted between the studies in this analysis, and the authors conducted a sensitivity analysis to confirm robustness. After excluding NOS score $<6,{ }^{25}$ the OR remained 0.34 vs. 0.36 . In a second subgroup analysis, the authors calculated a pooled OR for studies with retrospective case recruitment $(n=10)$ and prospective studies $(n=8)$. The retrospective group had an OR of 0.32 $(95 \% \mathrm{Cl}, 0.18-0.56)$, and the prospective group had an OR of $0.36(95 \% \mathrm{Cl}, 0.22-0.59)$. Finally, the authors excluded individual study estimates one at a time to examine the influence of each study on the overall OR. The omission of any one study did not appreciably change the pooled OR ( $O R=0.32-0.36$ ).

No potential publication bias was evident for the studies that evaluated bronchoscopy for patients with hemoptysis. The site of bleeding, massive hemoptysis, and publication bias could not be analysed due to the low number of studies. 


\begin{tabular}{|c|c|c|c|c|c|c|c|c|c|c|}
\hline \multirow{29}{*}{ A } & \multirow{2}{*}{$\begin{array}{l}\text { Study or Subgroup } \\
1.1 .1 \text { retro }\end{array}$} & \multicolumn{2}{|l|}{ FOB } & $\begin{array}{r}\mathrm{CT} \\
\text { Events }\end{array}$ & Total & \multicolumn{2}{|c|}{$\begin{array}{cc} & \text { Odds Ratio } \\
\text { Weight } & M-H, \text { Random. } 95 \% \mathrm{Cl}\end{array}$} & \multicolumn{3}{|c|}{$\begin{array}{l}\text { Odds Ratio } \\
\text { M-H, Random, } 95 \% \mathrm{Cl}\end{array}$} \\
\hline & & & & & & & & & & \\
\hline & Arooj-2018 (1) & $\begin{array}{r}31 \\
445\end{array}$ & $\begin{array}{r}102 \\
1089\end{array}$ & $\begin{array}{r}63 \\
845\end{array}$ & $\begin{array}{r}155 \\
1185\end{array}$ & $6.8 \%$ & $0.64[0.38,1.08]$ & & & \\
\hline & $\begin{array}{l}\text { christian-2020 } \\
\text { Fidan-2002 }\end{array}$ & $\begin{array}{r}445 \\
45\end{array}$ & $\begin{array}{r}1089 \\
52\end{array}$ & $\begin{array}{r}845 \\
77\end{array}$ & $\begin{array}{r}1185 \\
79\end{array}$ & $\begin{array}{l}7.6 \% \\
3.5 \%\end{array}$ & $\begin{array}{l}0.28[0.23,0.33] \\
0.17[0.03,0.84]\end{array}$ & $F$ & & \\
\hline & Hirshberg-1997 & 57 & 137 & 47 & 70 & $6.6 \%$ & $0.35[0.19,0.64]$ & & & \\
\hline & Khalil-2007 & 2 & 80 & 48 & 80 & $3.8 \%$ & $0.02[0.00,0.07]$ & $\longleftarrow$ & & \\
\hline & Mondoni-2019 & 237 & 487 & 241 & 545 & $7.5 \%$ & $1.20[0.94,1.53]$ & & - & \\
\hline & Naidich-1990 & 30 & 58 & 46 & 58 & $5.9 \%$ & $0.28[0.12,0.63]$ & & & \\
\hline & Nielsen-2016 & 54 & 326 & 174 & 326 & $7.3 \%$ & $0.17[0.12,0.25]$ & & & \\
\hline & Revel-2002 & 68 & 73 & 53 & 57 & $4.1 \%$ & $1.03[0.26,4.01]$ & & & \\
\hline & Seon-2016 & 84 & 161 & 125 & 161 & $7.0 \%$ & $0.31[0.19,0.51]$ & & & \\
\hline & Subtotal $(95 \% \mathrm{Cl})$ & & 2565 & & 2716 & $60.1 \%$ & $0.32[0.18,0.56]$ & & & \\
\hline & Total events & 1053 & & 1719 & & & & & & \\
\hline & $\begin{array}{l}\text { Heterogeneity: } \mathrm{Tau}^{2}= \\
\text { Test for overall effect: }\end{array}$ & $\begin{array}{l}0.68 ; \mathrm{Chi}^{2} \\
\mathrm{Z}=3.97(\mathrm{P}\end{array}$ & $\begin{array}{l}=138.9 \\
P<0.00\end{array}$ & $\begin{array}{l}5, d f=9( \\
01)\end{array}$ & $(P<0.0$ & $00001) ; 1^{2}=$ & $=94 \%$ & & & \\
\hline & 1.1.2 pros & & & & & & & & & \\
\hline & Abal-2001 & 14 & 46 & 35 & 41 & $5.0 \%$ & $0.07[0.03,0.22]$ & & & \\
\hline & Arooj-2018 (2) & 43 & 142 & 100 & 182 & $7.0 \%$ & $0.36[0.22,0.57]$ & & & \\
\hline & McGuinness-1994 & 45 & 57 & 50 & 57 & $5.2 \%$ & $0.53[0.19,1.45]$ & & & \\
\hline & Mohammad-2015 & 13 & 40 & 24 & 40 & $0.0 \%$ & $0.32[0.13,0.80]$ & & & \\
\hline & muhammad-2017 & 73 & 75 & 95 & 100 & $3.3 \%$ & $1.92[0.36,10.18]$ & & & \\
\hline & Patricia-1993 & 50 & 91 & 73 & 91 & $6.4 \%$ & $0.30[0.16,0.58]$ & & & \\
\hline & Tsoumakidou-2006 & 59 & 129 & 121 & 157 & $6.9 \%$ & $0.25[0.15,0.42]$ & & & \\
\hline & Uzun-2010 & 107 & $\begin{array}{l}124 \\
664\end{array}$ & 114 & 128 & $6.1 \%$ & $0.77[0.36,1.64]$ & & & \\
\hline & $\begin{array}{l}\text { Subtotal }(95 \% \mathrm{Cl}) \\
\text { Total events }\end{array}$ & 391 & 664 & 588 & 756 & $39.9 \%$ & $0.36[0.22,0.59]$ & & & \\
\hline & Heterogeneity: $\mathrm{Tau}^{2}=$ & $0.28 ; \mathrm{Chi}^{2}=$ & $=18.61$ & , df $=6(P$ & $P=0.00$ & $05) ; I^{2}=68$ & & & & \\
\hline & Total $(95 \% \mathrm{Cl})$ & & 3229 & & 3472 & $100.0 \%$ & $0.34[0.23,0.51]$ & & & \\
\hline & Total events & 1444 & & 2307 & & & & & & \\
\hline & Heterogeneity: $\mathrm{Tau}^{2}=$ & 0.55; $\mathrm{Chi}^{2}=$ & $=158.5$ & $1, d f=16$ & $0(P<0$. & $.00001) ; 1^{2}$ & $2=90 \%$ & 0.01 & 10 & 100 \\
\hline & $\begin{array}{l}\text { Test for overall effect: } \\
\text { Test for subaroun diffe }\end{array}$ & $\begin{array}{l}Z=5.27(P \\
\text { rences: } \mathrm{Ch}\end{array}$ & $\begin{array}{l}P<0.00 \\
h^{2}=0.16\end{array}$ & $\begin{array}{l}\text { 1001) } \\
0 . d f=1\end{array}$ & $(P=0.7$ & 75). $1^{2}=0 \%$ & & Favours [experimental] & Favours [control] & \\
\hline \multirow{30}{*}{ B } & Study or Subgroup & $\begin{array}{l}\text { FOE } \\
\text { Events }\end{array}$ & B Total & $\begin{array}{r}\text { CT } \\
\text { Events }\end{array}$ & Total & Weight & $\begin{array}{l}\text { Risk Difference } \\
\text { M-H, Fixed, } 95 \% \mathrm{Cl}\end{array}$ & \multicolumn{3}{|c|}{$\begin{array}{l}\text { Risk Difference } \\
\text { M-H. Fixed, } 95 \% \mathrm{Cl}\end{array}$} \\
\hline & Abal-2001 & 23 & 46 & 25 & 41 & $5.2 \%$ & $-0.11[-0.32,0.10]$ & & & \\
\hline & Khalil-2007 & 71 & 80 & 64 & 80 & $9.6 \%$ & $0.09[-0.02,0.20]$ & & & \\
\hline & Mohammad-2015 & 28 & 40 & 37 & 40 & $0.0 \%$ & $-0.23[-0.39,-0.06]$ & & & \\
\hline & Mondoni-2019 & 237 & 487 & 241 & 487 & $58.3 \%$ & $-0.01[-0.07,0.05]$ & & & \\
\hline & Revel-2002 & 53 & 73 & 40 & 57 & $7.7 \%$ & $0.02[-0.13,0.18]$ & & & \\
\hline & Seon-2016 & 107 & 161 & 105 & 161 & $19.3 \%$ & $0.01[-0.09,0.12]$ & & & \\
\hline & Total $(95 \% \mathrm{Cl})$ & & 847 & & 826 & $100.0 \%$ & $0.00[-0.04,0.05]$ & & & \\
\hline & Total events & 491 & & 475 & & & & & & \\
\hline & \multirow{2}{*}{\multicolumn{7}{|c|}{$\begin{array}{l}\text { Heterogeneity: } \text { Chi }^{2}=3.58, d f=4(P=0.47) ; I^{2}=0 \% \\
\text { Test for overall effect: } Z=0.09(P=0.93)\end{array}$}} & -1 & 0.5 & 1 \\
\hline & & & & & & & & Favours [experimental] & Favours [control] & \\
\hline & & FOB & & CT & & & Odds Ratio & & Ratio & \\
\hline & Study or Subgroup & Events & Total & Events & Total & Weight & M-H, Random, $95 \%$ CI & M-H, Rands & $10 \mathrm{~m} .95 \% \mathrm{Cl}$ & \\
\hline & Abal-2001 & 14 & 46 & 35 & 41 & $6.2 \%$ & $0.07[0.03,0.22]$ & & & \\
\hline & Fidan-2002 & 45 & 52 & 77 & 79 & $3.9 \%$ & $0.17[0.03,0.84]$ & & & \\
\hline & Hirshberg-1997 & 57 & 137 & 47 & 70 & $9.0 \%$ & $0.35[0.19,0.64]$ & & & \\
\hline & Khalil-2007 & 2 & 80 & 48 & 80 & $4.4 \%$ & $0.02[0.00,0.07]$ & + & & \\
\hline & McGuinness-1994 & 24 & 57 & 35 & 57 & $8.1 \%$ & $0.46[0.22,0.97]$ & & & \\
\hline & Mohammad-2015 & 13 & 40 & 24 & 40 & $7.1 \%$ & $0.32[0.13,0.80]$ & & & \\
\hline & Naidich-1990 & 22 & 58 & 39 & 58 & $8.0 \%$ & $0.30[0.14,0.64]$ & & & \\
\hline & Nielsen-2016 & 48 & 326 & 128 & 326 & $10.4 \%$ & $0.27[0.18,0.39]$ & - & & \\
\hline & Patricia-1993 & 39 & 91 & 55 & 91 & $9.1 \%$ & $0.49[0.27,0.89]$ & & & \\
\hline & Revel-2002 & 6 & 73 & 44 & 57 & $6.4 \%$ & $0.03[0.01,0.07]$ & 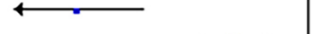 & & \\
\hline & Seon-2016 & 84 & 161 & 125 & 161 & $9.8 \%$ & $0.31[0.19,0.51]$ & & & \\
\hline & Tsoumakidou-2006 & 59 & 129 & 121 & 157 & $9.6 \%$ & $0.25[0.15,0.42]$ & $=$ & & \\
\hline & Uzun-2010 & 10 & 124 & 43 & 128 & $8.1 \%$ & $0.17[0.08,0.36]$ & & & \\
\hline & Total $(95 \% \mathrm{Cl})$ & & 1374 & & 1345 & $100.0 \%$ & $0.21[0.14,0.31]$ & & & \\
\hline & Total events & 423 & & 821 & & & & & & \\
\hline & Heterogeneity: $\mathrm{Tau}^{2}=$ & $=0.35 ; \mathrm{Chi}^{2}$ & $2=47.37$ & $7, \mathrm{df}=12$ & $(P<0 . C$ & $.00001) ; 1^{2}$ & $=75 \%$ & 0.01 & 10 & 100 \\
\hline & Test for overall effect & $Z=7.81$ & $(P<0.00$ & 0001) & & & & Favours [experimental] & Favours [control] & \\
\hline
\end{tabular}

Figure 3: Forest plots of utility compared bronchoscopy with CT (A), total (B), bleeding site, (C) bleeding cause. 


\begin{tabular}{|c|c|c|c|c|c|c|c|c|c|c|}
\hline Study or Subgroup & $\begin{array}{l}\text { FOB } \\
\text { Events }\end{array}$ & Total & $\begin{array}{r}\text { CT } \\
\text { Events }\end{array}$ & Total & Weight & $\begin{array}{l}\text { Odds Ratio } \\
\text { M-H, Fixed, } 95 \% \mathrm{Cl}\end{array}$ & & $\begin{array}{r}\text { Odds } \\
\text { M-H, Fixe }\end{array}$ & $\begin{array}{l}\text { Ratio } \\
\text { d, } 95 \% \mathrm{Cl}\end{array}$ & \\
\hline Abal-2001 & 0 & 9 & 1 & 9 & $1.3 \%$ & $0.30[0.01,8.35]$ & & & & \\
\hline McGuinness-1994 & 0 & 8 & 2 & 8 & $2.1 \%$ & $0.15[0.01,3.77]$ & & & & \\
\hline Naidich-1990 & 3 & 23 & 11 & 23 & $8.5 \%$ & $0.16[0.04,0.71]$ & & & & \\
\hline Patricia-1993 & 8 & 42 & 7 & 42 & $5.0 \%$ & $1.18[0.38,3.60]$ & & & & \\
\hline Tak-1999 & 5 & 50 & 17 & 50 & $13.6 \%$ & $0.22[0.07,0.64]$ & & & & \\
\hline Thirumaran-2009 & 36 & 269 & 88 & 257 & $69.4 \%$ & $0.30[0.19,0.46]$ & & & & \\
\hline Total $(95 \% \mathrm{Cl})$ & & 401 & & 389 & $100.0 \%$ & $0.32[0.22,0.45]$ & & & & \\
\hline Total events & 52 & & 126 & & & & & & & \\
\hline \multirow{2}{*}{\multicolumn{7}{|c|}{$\begin{array}{l}\text { Heterogeneity: } \mathrm{Chi}^{2}=6.83, \mathrm{df}=5(\mathrm{P}=0.23) ; \mathrm{I}^{2}=27 \% \\
\text { Test for overall effect: } Z=6.32(P<0.00001)\end{array}$}} & 0.0 & 0.1 & 10 & 100 \\
\hline & & & & & & & & [experimental] & Favours [control] & \\
\hline
\end{tabular}

Figure 4: Forest plots of utility compared bronchoscopy with CT when the radiograph is normal.

\begin{tabular}{|c|c|c|c|c|c|c|c|c|c|}
\hline Study or Subgroup & $\begin{array}{l}\text { FOB } \\
\text { Events }\end{array}$ & Total & $\begin{array}{r}\text { CT } \\
\text { Events }\end{array}$ & Total & Weight & $\begin{array}{c}\text { Odds Ratio } \\
\text { M-H, Random, } 95 \% \mathrm{Cl}\end{array}$ & $\begin{array}{r}\text { Odds } \\
M-H, \text { Rand }\end{array}$ & $\begin{array}{l}\text { s Ratio } \\
\text { dom. } 95 \% \mathrm{Cl}\end{array}$ & \\
\hline Hsiao-2001 & 26 & 28 & 13 & 13 & $25.0 \%$ & $0.39[0.02,8.77]$ & $=$ & & \\
\hline Khalil-2007 & 4 & 22 & 22 & 26 & $35.6 \%$ & $0.04[0.01,0.18]$ & & & \\
\hline Revel-2002 & 53 & 73 & 40 & 57 & $39.5 \%$ & $1.13[0.52,2.42]$ & & & \\
\hline Total $(95 \% \mathrm{Cl})$ & & 123 & & 96 & $100.0 \%$ & $0.27[0.02,3.18]$ & & & \\
\hline Total events & 83 & & 75 & & & & & & \\
\hline \multicolumn{7}{|c|}{$\begin{array}{l}\text { Heterogeneity: } \mathrm{Tau}^{2}=3.91 ; \mathrm{Chi}^{2}=14.88, \mathrm{df}=2(P=0.0006) ; I^{2}=87 \% \\
\text { Test for overall effect: } Z=1.05(P=0.29)\end{array}$} & $\begin{array}{ll}0.01 & 0.1 \\
\text { Favours [experimental] }\end{array}$ & $\begin{array}{lc}1 & 10 \\
\text { Favours [control] }\end{array}$ & 100 \\
\hline
\end{tabular}

Figure 5: Forest plots of utility compared bronchoscopy with CT in massive hemoptysis.

\begin{tabular}{|c|c|c|c|c|c|c|c|c|c|}
\hline Study or Subgroup & $\begin{array}{l}\text { FOB } \\
\text { Events }\end{array}$ & Total & $\begin{array}{r}\text { CT } \\
\text { Events }\end{array}$ & Total & Weight & $\begin{array}{l}\text { Odds Ratio } \\
\text { M-H, Fixed, } 95 \% \mathrm{Cl}\end{array}$ & $\begin{array}{l}\text { Odds } \\
\text { M-H, Fixe }\end{array}$ & $\begin{array}{l}\text { Ratio } \\
\text { d. } 95 \% \mathrm{Cl}\end{array}$ & \\
\hline Arooj-2018 (1) & 18 & 19 & 24 & 24 & $4.0 \%$ & $0.25[0.01,6.54]$ & 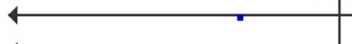 & & \\
\hline Arooj-2018 (2) & 25 & 28 & 33 & 33 & $9.1 \%$ & $0.11[0.01,2.20]$ & & & \\
\hline Lee-2012 & 1 & 1 & 0 & 1 & $0.3 \%$ & $9.00[0.10,831.78]$ & & & \\
\hline McGuinness-1994 & 6 & 7 & 7 & 7 & $3.5 \%$ & $0.29[0.01,8.39]$ & & & \\
\hline Mohammad-2015 & 2 & 2 & 2 & 2 & & Not estimable & & & \\
\hline Naidich-1990 & 19 & 24 & 24 & 24 & $13.2 \%$ & $0.07[0.00,1.39]$ & 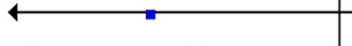 & & \\
\hline Nielsen-2016 & 8 & 13 & 12 & 13 & $11.3 \%$ & $0.13[0.01,1.36]$ & & & \\
\hline Patricia-1993 & 27 & 34 & 34 & 34 & $18.1 \%$ & $0.05[0.00,0.97]$ & $\vdash$ & & \\
\hline Revel-2002 & 6 & 9 & 9 & 9 & $8.2 \%$ & $0.10[0.00,2.23]$ & & & \\
\hline Seon-2016 & 3 & 4 & 4 & 4 & $3.3 \%$ & $0.26[0.01,8.52]$ & $\hookleftarrow$ & & \\
\hline Thirumaran-2009 & 14 & 26 & 24 & 24 & $28.9 \%$ & $0.02[0.00,0.43]$ & & & \\
\hline Total $(95 \% \mathrm{Cl})$ & & 167 & & 175 & $100.0 \%$ & $0.12[0.05,0.28]$ & & & \\
\hline Total events & 129 & & 173 & & & & & & \\
\hline \multicolumn{7}{|c|}{ Heterogeneity: $\mathrm{Chi}^{2}=5.81, \mathrm{df}=9(\mathrm{P}=0.76) ; \mathrm{I}^{2}=0 \%$} & 0.01 & 10 & 100 \\
\hline \multicolumn{7}{|c|}{ Test for overall effect: $Z=4.76(P<0.00001)$} & Favours [experimental] & Favours [control] & \\
\hline
\end{tabular}

Figure 6: Forest plots of utility compared bronchoscopy with CT in patients with a high risk of malignancy.

\section{DISCUSSION}

In this review, a meta-analysis of 4,635 subjects in 23 studies from PubMed, Embase, and the Cochrane Library was conducted to compare the utility of bronchoscopy and CT to determine the site and cause of hemoptysis along with the initial evaluation flow. It was found that bronchoscopy plays a less effective role in identifying the causes of hemoptysis than CT in any circumstance.

The strengths of the study include a comprehensive, systematic review of the literature and that the incidence and mortality of patients with hemoptysis was analysed and pooled accurately with appropriate epidemiologic methods. Jones reported that the annual incidence was approximately $0.1 \%{ }^{2,11}$ Among those patients, those with massive hemoptysis account for about $5-15 \%{ }^{21,31}$ and often come the emergency department at first. ${ }^{10}$ Therefore, the conclusion of this study could be useful in clinical practice.

Several diagnostic techniques are prescribed to assess hemoptysis. Chest radiography, bronchoscopy, and CT were the most frequently employed tools. For centuries, bronchoscopy has been considered the gold standard to detect bleeding sites. ${ }^{6}$

However, according to the findings and recent studies, chest 
CT and bronchoscopy may have similar utility in identifying the bleeding site $(\mathrm{OR}=0.00,95 \% \mathrm{Cl}$ : $[-0.04,0.05])$. A study by Revel et al. demonstrated equivalence between bronchoscopy and CT to localise the source of bleeding (73\% vs. 70\%, respectively). ${ }^{21}$ Indeed, given the development of highly sensitive imaging techniques ${ }^{1}$ combining the most ground-glass attenuation (GGA) with specific lesions, ${ }^{31} \mathrm{CT}$ demonstrated a remarkably higher ability to detect the exact location of bleeding than bronchoscopy both in early ([130/252 (51.6\%) vs. $73 / 190(38.4 \%), p=0.006)]$ and delayed examinations [(111/293 (37.9\%) vs. 65/261 (24.9\%)]. ${ }^{29}$ In a word, bronchoscopy did not show significant advantages than $\mathrm{CT}$ in identifying the source of hemoptysis.

Hemoptysis can be a sign of many different diseases varying from infections to malignancy. Effective evaluation flow is needed to identify the underlying pathogenesis so that the appropriate treatment can be employed. All the studies included in the review demonstrated that $\mathrm{CT}$, rather than bronchoscopy, should be applied to determine the types and causes of hemoptysis, including tumor, bronchiectasis, pneumonia, etc. Bronchoscopy is better used to identify the pathogen in infectious diseases, if the CT could not identify them accurately.

First, it is unnecessary to use bronchoscopy to screen each patient with hemoptysis (KQ1); second, when chest radiography is interpreted as normal, it is suggested that CT is superior to bronchoscopy (KQ 2). Nielsen et al. reported that the sensitivity on CT was $0.92(p<0.05)$, and the combination of FOB and computed tomography (CT) did not increase accuracy of diagnosis of malignant or nonmalignant causes in hemoptysis patients $(0.97, p=0.58){ }^{32}$

The only issue is the third key question (KQ 3); should bronchoscopy be used for patients with negative findings on CT. Indeed, there were many cryptogenic hemoptysis events, accounting for $5.4-83.8 \%$ of all hemoptysis events in the included studies and other published studies. This review showed that in 738 negative CT scans, only 4 cancers were detected by bronchoscopy based on two studies in 1997 and $2012 .^{18,24}$ In contrast, Petersen et al. retrospectively reviewed 1,185 patients in Denmark, with no malignancy suspected on computed tomography and no malignant disease by initial bronchoscopy. ${ }^{30}$ In 609 patients, lung cancer developed in $1.5 \%$ of patients $(n=9)$ in the following five years. ${ }^{14}$ In addition, bronchoscopists may need to intervene in iatrogenic bleeding since they perform procedures, such as transbronchial biopsies, which are associated with significant bleeding in $5.1-10.6 \%$ of cases. ${ }^{33,34}$ Bronchoscopy is only provided to limited patients due to limited medical resources. Therefore, patients without evidence of hemoptysis would likely benefit from an initial evaluation of bronchoscopy.

Additionally, it is recommended that in patients with hemoptysis, whose chest CT was negative for the cause of hemoptysis, a thorough, careful history and full examination were essential parts to distinguish from pseudohemoptysis. ${ }^{35}$ Among the 228 patients with hemoptysis and no identified cause on chest CT, Lee et al. found 43 cases that were not real hemoptysis. ${ }^{24}$ Savale et al. found anticoagulant and antiplatelet treatments predisposing patients to hemorrhage in $24 \%$ of their patients. $^{36}$

Massive hemoptysis represents one of the most challenging conditions in clinical practice. Severe hemoptysis accounts for only $10-15 \%$ of all hemoptysis cases, ${ }^{18}$ but is associated with a significant mortality rate as high as $80 \%$ without timely and effective management. There is no consensus on a uniform cutoff value for hemoptysis to be considered massive; the present inclusive studies reported 200, 300 and $400 \mathrm{ml}$ per 24 hours.

It is better to use the magnitude-of-effect definition, ${ }^{12,13}$ which rates the ability to clear tracheobronchial blood and impair lung function. ${ }^{1}$ Therefore, in this review, the author's definition was accepted.

Promptly identifying the location and cause of bleeding would vary depending on the condition of the patient. However, in this review, bronchoscopy used in patients with massive hemoptysis was not significantly different from CT $(95 \% \mathrm{Cl}$ : $[0.02,3.18]$ ) in general. Revel et al. reported that CT was comparable for identifying the bleeding site (70\% vs. $73 \%$ ) and much superior in determining the cause of bleeding $(77 \%$ vs. $8 \%){ }^{21}$ Similar results were reported in Khalil's studies (site: 80 vs. $88.8 \%$, cause: $60 \%$ vs. $2.5 \%){ }^{8}$ Indeed, the clinical focus on patients with massive hemoptysis is the rescue process rather than diagnosis. If the patient is relatively stable, bronchial artery embolisation (BAE) has been proven to be more effective in severe hemoptysis with reported immediate termination of bleeding rates from $70 \%$ to $99 \% .^{37-39}$ CT with IV contrast has been used with the intention of procedural planning for BAE, which was not discussed in our review. However, in unstable situations, it is preferable to secure the patient's airway before transfer to the operating room. Bronchoscopy could be performed at the bedside with an experienced bronchoscopic team and adequate equipment. Bronchoscopy may help clear the airways by aspirating or isolating the involved airway by selective endobronchial intubation and controlling the hemorrhage by using vasoconstrictive substances, or glue. ${ }^{40,41}$ Whether and when bronchoscopy should be determined according to the condition of the individual, but the only use for diagnosis is not needed.

Malignancy was one of the most frequent causes of hemoptysis with an incidence of $0.4-41.4 \%$, according to this review. A recent European observational study showed that malignancies were the most frequent etiology, ${ }^{42}$ and the majority of neoplasms were lung cancers $(106 / 116,91.3 \%)$ with endobronchial lesions (84/116, 72.4\%). Hemoptysis may be an early symptom of lung cancer, and a thorough investigation of patients with this symptom may lead to early diagnosis. ${ }^{43}$ The Danish Lung Cancer Group (DLCG) recommended in their guidelines that CT and bronchoscopy should be performed in patients who are smokers and 40 years of age or older. 
However, in this review, all the studies suggested that the application of initial bronchoscopy was futile in the detection of lung cancer compared to CT $(\mathrm{OR}=0.12)$. Petersen et al. reported that all cryptogenic patients with hemoptysis $(n=989)$ had no malignant disease by a prompt investigation, and lung cancer developed in $1.5 \%$ of patients in the following five years. ${ }^{30}$ This finding is comparable to results from previous studies. Tsoumakidou et al. found no new lung cancers in 189 hemoptysis patients during an average follow-up of 2.7 years. ${ }^{22}$ Bønløkke et al. studied 78 patients with no pathology on CT and found no malignancy within two years of initial referral. ${ }^{9}$ Additionally, Nielsen et al. reported that the sensitivity of detecting lung cancer by CT was 0.92 . Combining CT and bronchoscopy in these cases would not provide a better diagnostic yield given that the sensitivity was 0.97 , and the difference was insignificant $(p=0.58)$. Bronchoscopy did not identify any malignant etiologies not already diagnosed by $\mathrm{CT}^{32}$

Considering the possible complications and limited benefits of the procedure, it is reasonable that there is no need to perform direct bronchoscopy in all patients with hemoptysis. Moreover, due to likely underlying malignancies, there is a need for a dedicated follow-up of hemoptysis patients.

There are also certain limitations to this study. First, significant heterogeneity existed among the studies in this analysis. This finding is predictable given the presence of interstudy differences in study design (prospective and retrospective), enrolled populations with a wide spectrum of etiologies, and variations in the reported prevalence among different geographic locations. The heterogeneity among the studies remained despite the extraction of low-quality records, usage of a randomeffects model and subgroup analyses. Finally, the quantitative meta-analysis was performed based on secondary data, which may lead to inaccurate results.

\section{CONCLUSION}

This study suggested that bronchoscopy did not show superior diagnostic accuracy than CT for patients with hemoptysis at the first visit, particularly for those with normal radiography results. It is recommended that $\mathrm{CT}$ is firstly used rather than bronchoscopy at this circumstance. However, bronchoscopy could be used to further determine the pathology and pathogen cause. Each case with massive hemoptysis needs to be individually approached according to the patient's condition and team's abilities. Bronchoscopy should not be used exclusively for diagnosis.

\section{SOURCES OF SUPPORT:}

This study was supported by the National Natural Science Foundation of China (82072156) and the Science and Technology Department of Sichuan Province (2018JY0389, 2019YFS0443).

\section{CONFLICT OF INTEREST:}

The authors declared no conflict of interest.

\section{AUTHORS' CONTRIBUTION:}

$\mathrm{DH}, \mathrm{KH}, \mathrm{DH}$ : Conceived the study idea, designed the study and wrote the initial draft, collected the data and performed the statistical analyses.

ZL: Supervised the statistical analyses and writing.

All authors critically revised the manuscript for intellectual content and approved the final version.

\section{REFERENCES}

1. Olsen KM, Manouchehr-Pour S, Donnelly EF, Henry TS, Berry MF, Boiselle PM, et al. ACR appropriateness criteria ${ }^{\circledR}$ hemoptysis. J Am Coll Radiol JACR 2020; 17(5s):S148-s159. doi: 10.1016/j.jacr.2020.01.043.

2. Abdulmalak C, Cottenet J, Beltramo G, Georges M, Camus P, Bonniaud $\mathrm{P}$, et al. Haemoptysis in adults: A 5-year study using the French nationwide hospital administrative database. Eur Respiratory J 2015; 46(2):503-11. doi: 10.1183/09031936.00218214.

3. Tak S, Ahluwalia G, Sharma SK, Mukhopadhya S, Guleria R, Pande JN. Haemoptysis in patients with a normal chest radiograph: Bronchoscopy-CT correlation. Australasian Radiology 1999; 43(4):451-5. doi: 10.1046/j.1440-1673.1999.00712.x.

4. Fidan A, Ozdoğan S, Oruç O, Salepçi B, Ocal Z, Cağlayan B. Hemoptysis: A retrospective analysis of 108 cases. Respir Med 2002; 96(9):677-680. doi: 10.1053/rmed.2002.1359.

5. Arooj P, Bredin E, Henry MT, Khan KA, Plant BJ, Murphy DM, et al. Bronchoscopy in the investigation of outpatients with hemoptysis at a lung cancer clinic. Respiratory Med 2018; 139:1-5. Doi: 10,1016/j.rmed.2018.04.007.

6. Naidich DP, Lee JJ, Garay SM, McCauley DI, Aranda CP, Boyd $A D$. Comparison of CT and fiberoptic bronchoscopy in the evaluation of bronchial disease. American J Roentgenol 1987; 148(1):1-7. doi: 10.2214/ajr.148.1.1.

7. Uzun O, Atasoy Y, Findik S, Atici AG, Erkan L. A prospective evaluation of hemoptysis cases in a tertiary referral hospital. Clin Respiratory J 2010; 4(3):131-8. doi: 10.1111/j. 1752-699X.2009.00158.x.

8. Khalil A, Soussan M, Mangiapan G, Fartoukh M, Parrot A, Carette MF. Utility of high-resolution chest CT scan in the emergency management of haemoptysis in the intensive care unit: Severity, localisation and aetiology. British J Radiol 2007; 80(949):21-25. doi: 10.1259/bjr/59233312.

9. Bønløkke S, Guldbrandt LM, Rasmussen TR. Bronchoscopy in patients with haemoptysis and normal computed tomography of the chest is unlikely to result in significant findings. Danish Med J 2015; 62(8):A5123.

10. Vanni S, Bianchi S, Bigiarini S, Casula C, Brogi M, Orsi S, et al. Management of patients presenting with haemoptysis to a tertiary care italian emergency department: The florence haemoptysis score (FLHASC). Intern Emerg Med 2018; 13(3):397-404. doi: 10.1007/s11739-017-1618-8.

11. Jones R, Charlton J, Latinovic R, Gulliford MC. Alarm symptoms and identification of non-cancer diagnoses in primary care: Cohort study. BMJ (Clinical Research ed). 2009; 339:b3094. doi: 10.1136/bmj.b3094.

12. Sakr L, Dutau H. Massive Hemoptysis: An update on the role of bronchoscopy in diagnosis and management. Resp Intern Rev Thoracic Dis 2010; 80(1):38-58. doi: 10.1159/000 274492. 
13. Ibrahim WH. Massive haemoptysis: The definition should be revised. Eur Respir J 2008; 32(4):1131-2. doi: 10.1183/ 09031936.00080108.

14. Petersen $\mathrm{CL}$, Weinreich UM. Five-year follow-up of hemoptysis with no malignancy suspected on chest computed tomography: Recurrence, lung cancer and mortality. Eur Clin Respir J 2019; 6(1):1616519. doi: 10.1080/20018525. 2019.1616519.

15. Naidich DP, Funt S, Ettenger NA, Arranda C. Hemoptysis: CT-bronchoscopic correlations in 58 cases. Radiol 1990; 177(2):357-362. doi: 10.1148/radiology.177.2.2217769.

16. Set PA, Flower CD, Smith IE, Chan AP, Twentyman OP, Shneerson JM. Hemoptysis: Comparative study of the role of CT and fiberoptic bronchoscopy. Radiol 1993; 189(3):677680. doi: 10.1148/radiology.189.3.8234690.

17. McGuinness G, Beacher JR, Harkin TJ, Garay SM, Rom WN, Naidich DP. Hemoptysis: Prospective high-resolution CT/bronchoscopic correlation. Chest 1994; 105(4):1155-62. doi: 10.1378/chest.105.4.1155.

18. Hirshberg B, Biran I, Glazer M, Kramer MR. Hemoptysis: Etiology, evaluation, and outcome in a tertiary referral hospital. Chest 1997; 112(2):440-444. doi: 10.1378/chest.112.2.440.

19. Hsiao El, Kirsch CM, Kagawa FT, Wehner JH, Jensen WA, Baxter RB. Utility of fiberoptic bronchoscopy before bronchial artery embolization for massive hemoptysis. AJR American J Roentgenol 2001; 177(4):861-7. doi: 10.2214/ajr.177.4.1770861.

20. Abal AT, Nair PC, Cherian J. Haemoptysis: Aetiology, evaluation and outcome- a prospective study in a third-world country. Respiratory Med 2001; 95(7):548-552. doi: 10.1053/rmed.2001.1053.

21. Revel MP, Fournier LS, Hennebicque AS, Cuenod CA, Meyer G, Reynaud P, et al. Can CT replace bronchoscopy in the detection of the site and cause of bleeding in patients with large or massive hemoptysis? AJR American J Roentgenol 2002; 179(5):1217-24. doi: 10.2214/ajr.179.5.1791217.

22. Tsoumakidou M, Chrysofakis G, Tsiligianni I, Maltezakis G, Siafakas NM, Tzanakis N. A prospective analysis of 184 hemoptysis cases: Diagnostic impact of chest X-ray, computed tomography, bronchoscopy. Respir Int Review Thoracic Dis 2006; 73(6):808-814. doi: 10.1159/0000 91189.

23. Thirumaran M, Sundar R, Sutcliffe IM, Currie DC. Is investigation of patients with haemoptysis and normal chest radiograph justified? Thorax 2009; 64(10):854-6. doi: 10.1136/ thx.2008.108795.

24. Lee YJ, Lee SM, Park JS. The clinical implications of bronchoscopy in hemoptysis patients with no explainable lesions in computed tomography. Respir Med 2012; 106(3):413-9. doi: 10.1016/j.rmed.2011.11.010.

25. Davoodi M, Kordi M, Gharibvand MM, Shoushtari MH, Borsi H, Bahadoram M. Hemoptysis: Comparison of diagnostic accuracy of multi detector CT scan and bronchoscopy. Global J Health Sci 2015; 7(3):373-7. doi: 10.5539/gjhs.v7n3p373.

26. Seon HJ, Kim YH, Kwon YS. Localisation of bleeding sites in patients with hemoptysis based on their chest computed tomography findings: A retrospective cohort study. BMC
Pulmonary Med 2016; 16(1):160. doi: 10.1186/s12890016-0322-1.

27. Nielsen K, Gottlieb M, Colella S, Saghir Z, Larsen KR, Clementsen PF. Bronchoscopy as a supplement to computed tomography in patients with haemoptysis may be unnecessary. Eur Clin Respir J. 2016 Jun 23;3:31802. doi: 10.3402/ecrj.v3.31802. PMID: 27343164; PMCID: PMC 4920935.

28. Muhammad Shafeek KKVP, Jesin kumar C, Mohan V, Manoj DK, Rajani M. A prospective evaluation of hemoptysis cases in a tertiary referral Hospital. J Med Sci Clin Res 2017; 05(11):29813-18.

29. Mondoni M, Carlucci P, Cipolla G, Fois A, Gasparini S, Marani $S$, et al. Bronchoscopy to assess patients with hemoptysis: Which is the optimal timing? BMC Pulmonary Med 2019; 19(1):36. doi: 10.1186/s12890-019-0795-9.

30. Petersen $\mathrm{CL}$, Weinreich UM. Hemoptysis with no malignancy suspected on computed tomography rarely requires bronchoscopy. European Clin Respir J 2020; 7(1):1721058 doi: 10.1080/20018525.2020.1721058.

31. Jean-Baptiste E. Clinical assessment and management of massive hemoptysis. Crit Care Med 2000; 28(5):1642-1647. doi: 10.1097/00003246-200005000-00066.

32. Nielsen K, Gottlieb M, Colella S, Saghir Z, Larsen KR, Clementsen PF. Bronchoscopy as a supplement to computed tomography in patients with haemoptysis may be unnecessary. Eur Clin Resp J 2016; 3:318026. doi: 10.3402/ecrj.v3.31802.

33. Leiten EO, Martinsen EM, Bakke PS, Eagan TM, Grønseth R. Complications and discomfort of bronchoscopy: A systematic review. Eur Clin Respir J 2016; 3:33324. doi: 10.3402/ecrj.v3.33324.

34. Haga T, Cho K, Nakagawa A, Takagiwa J, Arakawa S, Sakamoto $Y$, et al. Complications of fiberoptic bronchoscopy in very elderly adults. J Am Geriatrics Soc 2016; 64(3):676-7. doi: 10.1111/jgs.13999.

35. Bhalla A, Pannu AK, Suri V. Etiology and outcome of moderate-to-massive hemoptysis: Experience from a tertiary care center of North India. Int J Mycobacteriol 2017; 6(3):307-10. doi: 10.4103/ijmy.ijmy_54_17.

36. Savale L, Parrot A, Khalil A, Antoine M, Théodore J, Carette $M F$, et al. Cryptogenic hemoptysis: From a benign to a lifethreatening pathologic vascular condition. Am J Respir Crit Care Med 2007; 175(11):1181-5. doi: 10.1164/rccm.200609-13620C.

37. Tom LM, Palevsky HI, Holsclaw DS, Trerotola SO, Dagli M, Mondschein J, et al. Recurrent bleeding, survival, and longitudinal pulmonary function following bronchial artery embolisation for hemoptysis in a US. Adult population. J Vascul Inter Radiol: JVIR 2015; 26(12):1806-13.e1801. doi: 10.1016/j.jvir.2015.08.019.

38. Ittrich H, Bockhorn M, Klose H, Simon M. The diagnosis and treatment of hemoptysis. Dtsch Arztebl Int 2017; 114(21):371-81. doi: 10.3238/arztebl.2017.0371.

39. Kathuria $H$, Hollingsworth $H M$, Vilvendhan R, Reardon $C$. Management of life-threatening hemoptysis. J Intensive Care 2020; 8(1):23. doi: 10.1186/s40560-020-00441-8.

40. Tüller C, Tüller D, Tamm M, Brutsche MH. Hemodynamic 
effects of endobronchial application of ornipressin versus terlipressin. Respiration 2004; 71(4):397-401. doi: 10.1159/000079646.

41. Chawla RK, Madan A, Aditya C. Glue in Hemoptysis. J Bronchol Interventional Pulmonol 2016; 23(4):e40-e42. doi: 10.1097/LBR.0000000000000304.

42. Mondoni M, Carlucci P, Job S, Parazzini EM, Cipolla G, Pagani
$M$, et al. Observational, multicentre study on the epidemiology of haemoptysis. European Respir J 2018; 51(1): 1701813. doi: 10.1183/13993003.01813-2017.

43. Mondoni M, Sferrazza Papa GF, Sotgiu G, Carlucci P, Pellegrino GM, Centanni S. Haemoptysis: A frequent diagnostic challenge. Eur Respir J 2016;47(1):348-50.doi:10.1183/ 13993003.01344-2015. 